\title{
Meta
}

Journal des traducteurs

Translators' Journal

\section{Processus mentaux étudiés chez des sujets allemands apprenant le français lorsqu'ils sont en train de traduire}

\section{Frank G. Königs et Reinhard Kaufmann}

Volume 41, numéro 1, mars 1996

Le(s) processus de traduction / Translation Process(es)

URI : https://id.erudit.org/iderudit/003341ar

DOI : https://doi.org/10.7202/003341ar

Aller au sommaire du numéro

Éditeur(s)

Les Presses de l'Université de Montréal

ISSN

0026-0452 (imprimé)

1492-1421 (numérique)

Découvrir la revue

Citer cet article

Königs, F. G. \& Kaufmann, R. (1996). Processus mentaux étudiés chez des sujets allemands apprenant le français lorsqu'ils sont en train de traduire. Meta,

41(1), 7-25. https://doi.org/10.7202/003341ar
Résumé de l'article

L'article ci-dessous rend compte d'une expérience au cours de laquelle des étudiants allemands d'études françaises devaient traduire un texte allemand en français tout en pensant à voix haute. L'expérience avait pour objectif d'obtenir des informations sur le processus de la traduction tel qu'il a lieu. A l'aide d'une série de paramètres, élaborés antérieurement, les données obtenues sont analysées. Les résultats se comprennent comme une contribution à une traductologie axée sur le processus même de la traduction, à une discipline, donc, dont l'objectif n'est pas la traduction telle qu'elle doit se dérouler, mais telle qu'elle se déroule effectivement. 


\title{
PROCESSUS MENTAUX ÉTUDIÉS CHEZ DES SUJETS ALLEMANDS APPRENANT LE FRANCCAIS LORSQU'ILS SONT EN TRAIN DE TRADUIRE.
}

\section{Résultats d'une petite étude de cas comme contribution à la didactique de la traduction et de l'enseignement des langues étrangères ${ }^{1}$}

Frank G. KönigS ET Reinhard KaUfManN Institut Herder, Université de Leipzig, Leipzig et Université de la Ruhr, Bochum, Allemagne

\begin{abstract}
Résumé
L'article ci-dessous rend compte d'une expérience au cours de laquelle des étudiants allemands d'études françaises devaient traduire un texte allemand en français tout en pensant à voix haute. L'expérience avait pour objectif d'obtenir des informations sur le processus de la traduction tel qu'il a lieu. À l'aide d'une série de paramètres, élaborés antérieurement, les données obtenues sont analysées. Les résultats se comprennent comme une contribution à une traductologie axée sur le processus même de la traduction, à une discipline, donc, dont l'objectif n'est pas la traduction telle qu'elle doit se dérouler, mais telle qu'elle se déroule effectivement.
\end{abstract}

\begin{abstract}
This article reports on an experiment in which German students of French translated a German text into French using a thinking-aloud protocol. The goal of the experiment was to collect data on the translation process as it actually occurs. Using a set of previously determined parameters, the authors analyze the data. Their findings are seen as contributing to a theory of the translation process itself and whose objective is translation not as it should take place, but as it actually takes place.
\end{abstract}

\section{INTRODUCTION}

La traductologie est en pleine évolution. Cette évolution s'est traduite par la parution, ces dernières années, d'un nombre croissant de travaux de recherche dans lesquels on ne se contente plus de suggestions à caractère normatif, quant à la question de savoir ce qui doit se passer lors de l'acte de traduction, mais où l'on attache beaucoup plus d'importance à saisir ce qui se passe effectivement. Outre les contributions contenues dans ce numéro, qu'il me soit permis de mentionner - à titre d'exemple et sans prétendre à l'exhaustivité — les travaux de Gerloff (1986), de Hönig (1988), de Königs (1986 ; $1987 ; 1990$ a), de Krings (1986), de Lörscher (1991), de Smith (1994) ou bien encore de Tirkkonen-Condit (1991). Indépendamment des questions relatives aux méthodes de recherche qui, bien sûr, peuvent être discutées dans ce cadre et qui commandent que l'on se garde de survaloriser l'impact des résultats obtenus, ces travaux modifient sensiblement le champ d'investigation de la traductologie. C'est pourquoi il n'y a rien d'étonnant à ce que de nouvelles contributions à ce champ d'investigation viennent consacrer la recherche relative au processus même de la traduction comme son élément le 
plus authentique (voir, par ex., Königs 1990b; Bell 1991; Stolze 1994). Par contre, il apparaît plutôt étonnant que ce recours au phénomène même de la traduction reste ignoré lors de la description du phénomène qui consiste à traduire - ceci vraisemblablement plutôt en raison d'une croyance à caractère normatif que d'une argumentation fondée (c'est ce que fait, par ex., Newmark (1991) dans une large mesure, ainsi que Koller dans l'ouvrage Einführung in die Übersetzungswissenschaft, par ailleurs à recommander vivement, qui ne fait qu'effleurer ce domaine dans la quatrième édition remaniée de 1992).

Il n'y a aucun doute que cette orientation vers le processus même de la traduction en est encore à ses débuts et il serait déplacé de vouloir déjà simuler, dans cette phase initiale de la recherche, l'assurance qui est propre à une phase terminale. Nous en sommes encore très loin. Toutefois, il paraît nécessaire d'accorder à cet aspect toute l'attention qui lui est dû, étant donné qu'il est légitime d'attendre de cette recherche orientée vers le processus même de la traduction des conclusions fondées quant au phénomène en soi, ainsi que par rapport à l'enseignement et à l'apprentissage de la traduction. L'étude qui va suivre se comprend comme une modeste contribution dans ce domaine.

Par conséquent, l'objectif de notre étude consistera à éclairer la traduction en tant que processus mental. La traductologie, discipline relativement jeune, s'efforçait, jusqu'à présent, de développer essentiellement des procédés généraux de résolution des difficultés, procédés s'effectuant pas à pas et dans un certain ordre, cela afin de structurer le processus de la traduction et, ainsi, d'élaborer des recommandations à l'attention du traducteur.

Les représentants de la traductologie ont élaboré un grand nombre de modèles différents censés illustrer le processus de la traduction. Le grand défaut commun à tous ces modèles est le suivant: ils ne sont pas d'une grande aide pour le traducteur. Ils se bornent en effet tout simplement à décrire de façon très simplifiée ce qui devrait se passer, idéalement, au cours de la traduction. Cependant, ils ne décrivent ni n'expliquent le processus de la traduction pour ainsi dire, qui est, de par sa nature même, un processus mental. Les modèles élaborés par la traductologie traditionnelle, et traitant du processus de la traduction, n'énoncent, pour utiliser une formule polémique, rien d'autre que des lieux communs. Pendant longtemps, la traductologie ne s'est guère préoccupée de ce qui se passait effectivement dans la tête de la personne en train de traduire.

Or, ce sont précisément ces processus mentaux qui constituent l'intérêt proprement dit du mécanisme de la traduction. De ce point de vue, une approche orientée vers la psycholinguistique part du principe que le processus de traduction est un processus mental individuel et que chaque traducteur suit le chemin qui lui est propre pour atteindre son objectif. Le présent article a pour objet d'apporter une contribution à l'investigation de ce cheminement, qui ne se laisse vraisemblablement pas représenter par des modèles à caractère général.

La présente étude ne vise pas le traducteur professionnel: elle s'intéresse, au contraire, aux activités mentales de trois étudiants en français de niveau avancé, lorsque ces derniers sont en train de traduire. Naturellement, on peut objecter qu'il s'agit d'un public totalement différent de celui habituellement visé par la traductologie, ce qui est évidemment exact. Ce sont des traducteurs non professionnels que nous avons choisi d'étudier, et rien ne justifie l'hypothèse que les processus mentaux relevés chez les sujets de l'expérience ressemblent à ceux que l'on pourrait observer chez des traducteurs professionnels. Quoi qu'il en soit, les sujets de l'expérience traduisent et se livrent donc, par le fait même, et indépendamment de la qualité du produit de leur traduction, à une activité que l'on peut qualifier de traductrice. 
L'ambition de cette étude — et d'autres études analogues - consiste moins à dégager de nouvelles idées concernant la traduction comme activité professionnelle qu'à ouvrir de nouvelles perspectives quant aux activités mentales intervenant lorsque des sujets - en train de perfectionner à la fois leurs compétences linguistiques et leur compétence en matière de traduction - traduisent. Même si une équation entre ces processus ainsi mis à jour et ceux propres aux traducteurs professionnels n'est pas défendable, il existe cependant, dans une large mesure, des points communs entre les sujets analysés ici et les étudiants qui se trouvent en cours de formation pour devenir traducteurs, que l'on ne peut nier a priori. C'est pourquoi l'étude présentée ici s'interprète tout particulièrement comme une contribution qui concerne les activités mentales intervenant lors de la constatation de difficultés de traduction, et ce, dans le cadre de l'amélioration d'une compétence en langue étrangère et comme contribution à la didactique de la traduction.

\section{ORGANISATION ET RÉALISATION DE L'EXPÉRIENCE}

Pour obtenir de nouvelles données sur les processus mentaux lors de la réalisation d'une traduction ainsi que des données susceptibles d'être comparées à celles de travaux antérieurs, l'expérience a été réalisée avec trois sujets ayant chacun pour tâche de traduire un texte. Étant donné que les processus mentaux se dérobent à l'observation directe, on a eu recours, pendant l'expérience, au procédé d'introspection de la pensée à voix haute emprunté à la psychologie. Celui-ci doit permettre d'appréhender ce qui se passe dans la tête du sujet lorsqu'il se trouve confronté à la recherche de la solution d'une difficulté. L'hypothèse préalable fondamentale et essentielle sur laquelle s'appuie ce procédé est la théorie du traitement de l'information dans la mémoire de l'être humain.

... das informationsverarbeitende Individuum nimmt neue Informationen in der Weise auf, daß es sie in seinen vorhandenen Wissensstand einpaßt und dazu die verfügbaren Informationen abruft. Dieses Abrufen läuft über die beiden Arten des Gedächtnisses, nämlich das Kurzzeit- und das Langzeitgedächtnis. Fest internalisierte Informationen sind dieser Annahme zufolge - im Langzeitgedächtnis gespeichert. Sollen diese Informationen ergänzt werden, dann müssen sie zunächst in das Kurzzeitgedächtnis geholt werden, um abrufbar zu werden. Dort können sie dann auch weiterverarbeitet werden und darüber hinaus - das ist für unseren Zusammenhang wichtig - zumindest in Teilen verbalisiert werden. Wenn man also die Aufgabe so wählt, daß diese Verbalisierungen Bestandteil der Aufgabe werden, erhält man Informationen über die Art und Weise, wie Informationen verarbeitet werden. (Königs 1989 : 154)

Ainsi, dans notre cas, les sujets de l'expérience avaient pour tâche de traduire un texte et de verbaliser tout ce qui leur venait à l'esprit. Toutefois, et c'est aussi ce qu'ont montré les pauses au cours de l'expérience, une verbalisation complète des processus de traitement mentaux n'est guère réalisable. Un facteur qui influence considérablement le résultat de l'expérience est le degré de familiarité du sujet de l'expérience avec la pratique de la pensée à voix haute. Dans l'expérience présente, on a donné aux trois sujets - qui eurent à pratiquer pour la première fois la pensée à voix haute - le même texte allemand à traduire en français. Les trois sujets, dont la langue maternelle est l'allemand, étaient des étudiants de second cycle de français à l'Université de la Ruhr, à Bochum. Tous les trois avaient déjà réussi l'examen de traduction de fin d'études. Le texte qui leur fut soumis était délibérement plus court et moins difficile que ne le sont en général les textes de l'examen de fin d'études. Ce choix visait, d'une part, à restreindre la richesse du matériel à évaluer à une quantité de données maîtrisables et, d'autre part, à ne pas rebuter a priori les sujets de l'expérience par un degré de difficulté trop élevé. Le texte à traduire, ainsi que la traduction modèle proposée, étaient empruntés à un manuel d'exercices de 
traduction comprenant des textes allemands à traduire en français, à savoir : Gardes/Lacourière/Masqueliez, Lösungsheft zum Übersetzungskurs für Anfänger und Fortgeschrittene (Deutsch-Französisch), $2^{\mathrm{e}}$ éd., Borken, 1983.

Contrairement à ce qui a cours aux examens de traduction subis pendant leurs études ou lors de l'examen final, les sujets de l'expérience avaient à leur disposition des dictionnaires et des grammaires. Cela nous semblait être une démarche sensée, dans la mesure où l'objectif visé par la traduction imposée n'était pas la sélection, mais l'obtention de nouvelles données relatives à ce qui se passe dans la tête des sujets de l'expérience au cours de leur confrontation à la traduction. Ce faisant, la question de savoir quand et comment les sujets de l'expérience avaient recours aux divers outils mis à leur disposition n'était pas non plus dénuée d'intérêt. Par ailleurs, aucun traducteur professionnel ne travaille sans outils de référence. Les ouvrages mis à la disposition des sujets de l'expérience étaient les suivants :

WEIS, Erich (1985) : Pons-Globalwörterbuch/Weis ; Mattutat, Teil 1, französisch-deutsch, $2^{\mathrm{e}}$ éd., Stuttgart.

WEIS, Erich (1985) : Pons-Globalwörterbuch/Weis ; Mattutat, Teil 2, deutsch-französisch, $2^{\mathrm{e}}$ éd., Stuttgart.

ROBERT, Paul (1985) : Le Petit Robert 1. Dictionnaire alphabétique et analogique de la langue française, Paris, Le Robert.

KLEIN, Hans-Wilhelm et KLEINEIDAM, Hartmut (1983): Grammatik des heutigen Französisch, Stuttgart.

GREVISSE, Maurice (1953): Le bon usage. Cours de grammaire française et de langage français, $5^{\mathrm{e}}$ éd., Paris, Duculot.

ARRIVÉ, Michel et al. (1984): Grammaire Larousse du français contemporain, Paris, Larousse.

Pendant que les sujets de l'expérience traduisaient le texte qui leur était soumis en pensant à voix haute, un magnétophone enregistrait leurs paroles. Lorsqu'ils avaient recours aux outils mis à leur disposition, cela était, chaque fois, consigné par l'expérimentateur. Aucun délai n'était imposé, afin de permettre aux sujets d'utiliser le temps qu'il leur fallait, besoin qui se trouvait bien entendu élargi du fait de l'utilisation d'outils de référence. Voilà qui constitue naturellement une différence fondamentale entre l'expérience et les conditions imposées en situation d'examen, qu'il s'agisse d'examens partiels ou finaux.

Par la suite, on a transcrit les enregistrements de ce qui avait été pensé à voix haute. Les transcriptions consignaient exactement les moments où a eu lieu l'utilisation des outils. Elles feront l'objet d'une évaluation en fonction de certains critères et seront données en annexe.

\section{ÉVALUATION DE L'EXPÉRIENCE}

Bien que le texte à traduire soit relativement court et de difficulté moyenne, la transcription du matériel nous livre énormément de données. C'est pourquoi nous nous contenterons ici de ne présenter, à titre d'exemple, que quelques résultats de l'expérience. Une évaluation complète des données présentées en annexe - pour le sujet A - fera l'objet de travaux ultérieurs.

\section{Critères d'évaluation}

L'évaluation du matériel constitué par les données recueillies se fera selon les critères déjà élaborés par Königs (1989) - ceci tant pour obtenir des résultats relatifs à 
la mise à nu des processus mentaux que pour pouvoir les comparer. Ces critères sont les suivants :

1. Macro-contextualisation : on entend par là toutes les verbalisations se rapportant à l'organisation du texte dans son ensemble et signalant que le sujet avait bien en vue l'organisation de la totalité du texte.

2. Les étapes de l'agencement : ce sont les transformations concrètes effectuées successivement par le sujet en vue de mener à bien son travail de contextualisation.

3. Énonciation et solution des problèmes : ces deux notions désignent respectivement tous les phénomènes identifiés par le sujet comme des problèmes concrets, ainsi que les tentatives de solution succédant à leur identification.

4. Orientation linguistique : dans cette rubrique on indique si les tentatives de solution prennent appui sur la langue maternelle ou sur la langue étrangère, c'est-à-dire si le sujet s'oriente plutôt vers la langue maternelle ou vers la langue étrangère pour parvenir à la solution d'un problème.

5. Associations : quelles associations sont déclenchées par un élément de la langue étrangère et dans quelle mesure contribuent-elles à la solution d'un problème ?

6. Corrections : il s'agit ici de savoir quelles sont les rectifications que le sujet fait subir à son texte, quand et comment il y procède.

7. Utilisation du dictionnaire : ici, on observe comment les sujets travaillent avec le dictionnaire, quelles solutions ils choisissent par opposition aux autres possibilités qui s'y trouvent consignées et, surtout, comment ils justifient leur choix.

8. Transfert culturel : on retiendra sous cette notion à quels passages et avec quelle motivation concrète les sujets perçoivent et transforment les concepts linguistiques (existant dans leur langue maternelle) en fonction de la culture étrangère.

9. Pauses : toutes les pauses de deux secondes ou davantage faites au cours de la verbalisation ont été consignées. Elles permettent d'une part des déductions pour savoir si les sujets ont tenté - consciemment ou non - de se soustraire aux impératifs de l'expérience et, d'autre part, elles signalent surtout avec quel poids cognitif certains problèmes sont traités. Les analyses des pauses constituent donc autant de supports pour l'interprétation des données.

\section{RÉSULTATS}

La macro-contextualisation n'est pas très prononcée chez les sujets de l'expérience. Elle a lieu - quand c'est le cas - avant que les sujets ne s'attaquent à la traduction même. D'abord, pour se faire une première idée de ce qui les attend, ils commencent tous par une lecture complète du texte allemand qu'ils ont à traduire.

Tandis que le sujet $B$ ne fait que survoler succinctement le texte (et malheureusement pas à voix haute ; probablement a-t-il quelques difficultés initiales à s’habituer à penser tout haut !) et commence ensuite tout simplement à traduire jusqu'à ce qu'il se heurte à la première difficulté, on peut reconnaître chez le sujet $\mathrm{A}$ une contextualisation relative à l'organisation de l'ensemble du texte qui lui est soumis, et ce, en ce qui concerne le choix des temps du passé, comme le montre l'exemple suivant:

Einmal im Winter, an einem stürmischen Abend, klopfte es an der Tür (2) - passé simple. [...] Er kam oft vorüber, klopfte und hielt mir die Hand entgegen (3) Hintergrundbeschreibung, also imparfait. Nie sagte er ein Wort des Grußes oder des Dankes, er sah mich nur an mit seinen wäßrigen Trinkeraugen, und ich gab ihm, was mir einfiel, ein Endchen Wurst oder etliche Groschen aus der Hosentasche - weiterhin imparfait.

Sans aucun doute le sujet $\mathrm{A}$ sait déjà par expérience que, d'une part, le choix des temps du passé constitue pour lui une difficulté particulière, et que d'autre part ces temps du 
passé jouent un rôle important dans les textes narratifs. Aussi sa macro-contextualisation se concentre-t-elle sur ce problème ; elle est donc, dans le cas présent, induite par l'expérience et par l'apprentissage, et ce, sans équivoque : le sujet $\mathrm{A}$ reprend des pratiques empruntées à son apprentissage dans les cours de traduction. Après avoir lu une fois le texte en entier, après avoir chaque fois réfléchi au temps du passé à choisir, il décide de commencer à traduire. Par la suite, il ne prend pour ainsi dire plus en considération le texte dans son ensemble ; bien au contraire, il fait avancer sa traduction phrase par phrase.

On peut également déceler une macro-organisation chez le sujet $C$ lorsqu'il procède à une première lecture du texte, toutefois il s'agit plutôt d'une ébauche de macrocontextualisation. Celle-ci concerne aussi bien des difficultés d'ordre lexical que des difficultés relatives au choix des temps du passé. En outre, le sujet $\mathrm{C}$ se fait dès le départ des idées à propos du degré de difficulté du texte.

Nie sagte er ein Wort des Grußes oder des Dankes, er sah mich nur an mit seinen wäßrigen Trinkeraugen, (2) hm, das müßt' ich gleich mal nachgucken, (2) und ich gab ihm, was mir einfiel, ein Endchen Wurst oder etliche Groschen aus der Hosentasche. [...] Da nahm ich meine wollene Haube vom Haken, ach du lieber Gott (2), ein wenig schwankte der Alte, als ich ihm die Mütze über die Ohren zog, und dann ging er wortlos davon. Das aber war der Augenblick, in dem ich mich hätte besinnen müssen. (3) Jetzt geht die Handlung los. [...] Und da schlug ich die Tür zu. (2) Ja, das wird ja witzig, (2) schwieriger Text.

Par la suite, on ne décèle plus chez le sujet $\mathrm{C}$ quelque activité de contextualisation que ce soit relative à l'ensemble du texte. L'unité de contextualisation reste chez lui (tout comme chez les sujets $A$ et $B$ ) la phrase prise isolément voire un syntagme. Le sujet $C$ explique même expressément ce procédé qui lui est familier :

\section{So, jetzt werd' ich das dann Satz für Satz übersetzen, so wie ich das sonst auch immer mach'.}

Les trois sujets de l'expérience, et cela constitue un dénominateur commun qui saute aux yeux, ne réalisent une macro-contextualisation qu'avant de se lancer dans la traduction à proprement parler. La mise en œuvre concrète nécessaire à la réalisation de l'activité de contextualisation, avant tout la vérification de mots de vocabulaire inconnus, n'a lieu que lorsque l'unité de traduction (phrase ou syntagme) à traduire apparaît.

Il est caractéristique des étapes de l'agencement que les sujets de l'expérience se lancent d'abord dans la traduction et ne s'arrêtent que lorsqu'ils se heurtent à une difficulté. En voici un exemple :

Einmal im Winter, an einem stürmischen Abend, klopfte es an der Tür. (3) Une fois, (2) hm, une fois kann man für einmal glaub' ich so nicht benutzen, (3) was kann man da nehmen? (6) Ja, un jour könnte man nehmen, aber dann paßt das mit dem stürmischen Abend nicht. (14) ich schau' mal nach, aber ich fürchte, da werd' ich nicht viel finden bei einmal (schaut nach im deutsch-französischen Wörterbuch); einmal - ja, da steht une fois; (liest weiter); einst - autrefois, jadis, das kann man nicht nehmen; (13) hm, ja, was machen wir denn da? (2) Ja, nehmen wir halt mal dieses une fois, obwohl ich mir da nicht sicher bin; also une fois, (2) en hiver, (5) lors d'une soirée, (6) hm, stürmisch, stürmisch, (2) nee, das muß ich nachschlagen..

Il est frappant de voir ici qu'après avoir lu une fois toute la phrase, le sujet $\mathrm{C}$ ne fait qu'une pause de trois secondes avant de se lancer spontanément dans la traduction et ne met en doute que par la suite cette solution (correcte !).

Le procédé, consistant à lire systématiquement tout d'abord une fois jusqu'au bout chaque phrase à traduire et à commencer ensuite la traduction de façon relativement spontanée jusqu'à ce que la première difficulté apparaisse, est adopté d'un bout à l'autre de l'expérience par les trois sujets. La traduction a alors parfois même lieu mot à mot, tout particulièrement lorsque les difficultés rencontrées sont d'ordre lexical : 
J'imaginai la manière dont ce type (3) laisserait (...) tomber seine Fetzen, (3), tja, ses vêtements könnte man natürlich nehmen, das trifft den Sinn zumindest, aber für Fetzen direkt gibt es auch bestimmt noch was Besseres, ich schlag' man nach (schlägt im deutsch-französischen Wörterbuch nach); Fetzen - lambeau, chiffon; Lumpen - haillon, guenille; (2) haillon ist gut, ses haillons; la manière dont ce type laisserait tomber ses haillons (3) sur le sol, (2) sur le sol gewachst, hm, gewachst muß ich auch nachschlagen (sieht nach im deutsch-französischen Wörterbuch); wachsen — cirer, ach ja, sur le sol ciré dann also,...

Les pauses d'une certaine longueur précédant la traduction sont extrêmement rares. Les sujets traduisent plutôt d'une manière plus ou moins spontanée au moins une unité de traduction, si petite soit-elle, et même si ce n'est que le premier mot de la phrase qu'ils abordent. Au cours même de la traduction, leur contextualisation ne s'effectue même pas au niveau de la phrase, et ceci surtout pour les phrases d'une certaine longueur. Cela correspond vraisemblablement chez les sujets à un besoin de traduire tout d'abord jusqu'au point où ils se sentent capables de le faire avant de s'arrêter à une difficulté. Si, dès le départ, ils se consacraient, au début de chaque phrase, à ce qui leur pose problème, peut-être ressentiraient-ils la difficulté comme insurmontable. Au lieu de quoi, ils préfèrent écrire immédiatement tout ce qu'ils savent ou croient savoir.

Lorsqu'ils rencontrent une difficulté, ils l'expriment d'une façon plus ou moins claire. Outre des pauses parfois assez longues qui - si l'on part du principe que les sujets ne se soustraient pas aux règles de l'expérience - indiquent que ces derniers sont en train de chercher des informations dans la mémoire à long terme, des interjections, comme $h m$ ou tja (en allemand), sont presque toujours exprimées ensuite. Mais le plus souvent, les difficultés se trouvent explicitement énoncées.

Les tentatives concrètes de solution se trouvent donc en général précédées de l'éclaircissement explicite de la difficulté rencontrée :

Bei solchem Wetter lasse ich gern das Licht vor dem Haus brennen, damit mir die Nacht nicht zu nah an die Fenster kommt. (8) Jetzt stellt sich die Frage, wie man bei solchem Wetter übersetzt; (20) hm, puh, (15), tja, bei solchem Wetter, (13), ja, ich greif' jetzt am besten noch mal auf (5), ja, daß es stürmisch ist (12), quand il fait orageux (3), ja, das trifft den Sinn schon;...

Les pauses relativement longues indiquent ici que le sujet $B$ cherche longtemps dans sa mémoire à long terme l'expression française correspondant à la structure allemande bei solchem Wetter. Comme il ne trouve pas l'équivalent «par un temps pareil», il finit par se décider pour une périphrase qui a au moins le mérite de rendre compte du sens de l'énoncé de la langue de départ. En construisant en français une subordonnée temporelle, il complique, du point de vue linguistique, la structure par rapport au texte allemand mais grâce à cette complexité accrue due à une autre formulation, la solution du problème est devenue possible.

Chez le sujet $\mathrm{C}$ également, on trouve un exemple d'une structure plus complexe choisie pour la traduction par rapport au texte de la langue de départ; toutefois, cette stratégie n'aboutit pas dans le cas présent à une solution acceptable :

Nun ging ich also verdrossen, um nach diesem späten Gast zu sehen. $[. . . \mathrm{hm}$, verdrossen, das müßt' ich noch mal nachsehen $1 . .$. J (schlägt im deutsch-französischen Wörterbuch nach); verdrossen - fâché, nee fâché paßt nicht, das heißt ja eher wütend; (liest weiter) contrarié, renfrogné, dépité; (5) ja nee, fâché ist auf jeden Fall zu stark; (7) das Problem ist natürlich jetzt auch, daß wir hier alles Partizipien haben, und die kann ich so schlecht als Adverb nehmen, (5) da trenn' ich das am besten mit Komma ab und mach' das mit de façon, (3) dann brauch' ich mir keine Sorgen mehr zu machen; also, alors j'y allais, de façon (3) contrariée... 
Certes, le sujet $\mathrm{C}$ a raison en identifiant la difficulté, à savoir que l'on ne peut pas utiliser un participe passé comme adverbe; cependant, la stratégie souvent possible d'avoir recours à la traduction par 'de façon + adjectif' est fausse dans le cas présent étant donné que le participe verdrossen rencontré dans le texte de départ n'est pas utilisé adverbialement et ne se rapporte pas à l'expression verbale ich ging, mais plutôt à l'humeur du narrateur à la première personne. Ainsi le sujet $\mathrm{C}$ ne parvient pas à la solution qui consiste à séparer tout simplement l'adjectif «contrarié» par deux virgules et à le mettre ainsi en apposition au sujet «je».

On notera toutefois que les difficultés les plus thématisées, et de loin, par les sujets de l'expérience sont d'ordre lexical. Le sujet $\mathrm{C}$ va même jusqu'à porter de façon explicite un jugement sur le degré de difficulté du texte :

[...]. Na, wenn ich da kein Wörterbuch hätte, wüßt' ich ja überhaupt nichts dazu zu schreiben.

À cet endroit, après avoir de nouveau lu entièrement le deuxième paragraphe du texte de départ et avant d'en commencer la traduction, le sujet $\mathrm{C}$ procède encore une fois à une sorte de macro-contextualisation en portant un jugement sur le degré de difficulté de tout le texte, et ce, en s'appuyant sur les difficultés d'ordre lexical rencontrées. Il semble donc que, d'une manière générale, un texte contenant de nombreux mots de vocabulaire inconnus apparaisse a priori comme difficile, et ce, indépendamment des difficultés grammaticales qu'il recèle. Lors de la lecture du texte, ces dernières ne sautent pas aux yeux autant que les mots inconnus.

L'exemple suivant, mettant l'accent sur l'identification de la difficulté rencontrée, est digne d'intérêt dans la mesure où le sujet de l'expérience attribue son problème lexical à l'expression utilisée dans la langue de départ :

...das frische Leintuch, was heißt denn ein Leintuch? (3) Komische Sprache hier, ein Leintuch, (2) muß ich auch schon wieder nachgucken (sicht im deutsch-französischen Wörterbuch nach); Leintuch - drap, ach ja, na klar, hätt' ich auch drauf kommen können, (2) wär' ich auch drauf gekommen, wenn da Bettlaken statt Leintuch gestanden hätte,...

La critique à l'égard du texte de départ et - après la réussite dans la résolution de la difficulté grâce à l'aide apportée par le dictionnaire - l'autoconfirmation exprimant qu'il aurait, bien entendu, su la traduction de «drap» si, dans le texte allemand, il y avait eu le mot Bettlaken, aide le sujet $\mathrm{C}$ à compenser en quelque sorte le sentiment de frustration ressenti. Il est soulagé de pouvoir ultérieurement attribuer la difficulté lexicale rencontrée au texte de départ.

De nombreuses autres stratégies aboutissant à la solution du problème se laissent facilement démontrer, toutefois cela dépasserait le cadre de cette étude. Cependant, un autre procédé important permettant la solution du problème et auquel tous les sujets ont souvent eu recours, à savoir l'utilisation du dictionnaire, fera plus loin l'objet de considérations plus approfondies.

La tâche qui consiste à traduire un texte allemand en français conditionne l'orientation linguistique des tentatives de solution, à savoir, celles-ci s'effectuent majoritairement dans la langue maternelle. Les sujets s'orientent donc plutôt vers leur langue maternelle pour résoudre les problèmes que pose la traduction. Cela est tout à fait naturel puisque, dans le cas présent de la tâche à exécuter, c'est au niveau de la langue maternelle que se situe le déclic provoquant l'apparition d'une difficulté. On rencontre toutefois quelques rares cas où les sujets s'orientent vers la langue étrangère pour trouver une solution. En voici un exemple :

..., j' imaginai la manière (2) dont (4) dieser Kerl, (2) ja, ce mec geht wohl nicht, da muß ich mal nachschlagen, was hier noch möglich ist (schlägt nach im deutsch-französischen 
Wörterbuch); Kerl - homme, individu, garçon, gaillard, drôle, lascar, type, bougre (hält inne); type, ja genau, type, das gefällt mir am besten; (4) oder doch gaillard? (3) Hm, aber das ist ja eher so ein munterer Bursche wohl; nein, nehmen wir type, das ist neutraler;...

Après avoir consulté le dictionnaire allemand-français pour résoudre la difficulté, le sujet $\mathrm{C}$ entreprend une tentative de solution orientée vers la langue étrangère afin de pouvoir choisir parmi les différentes propositions du dictionnaire. Comme, de toute évidence, il sait que l'adjectif «gaillard» en allemand signifie quelque chose comme munter, il suppose, à juste titre, derrière le substantif ayant la même consonance une notion correspondante et se décide finalement pour le substantif plus neutre type. Il est à noter que l'on rencontre d'ailleurs assez souvent chez le sujet $C$ une orientation vers la langue étrangère dans ses tentatives de solution lorsqu'il doit choisir parmi les diverses propositions que lui offre le dictionnaire allemand-français. Alors, il consulte ensuite parfois le dictionnaire unilingue français pour vérifier si un mot convient mieux qu'un autre au présent contexte. Cette façon de procéder n'apparaît pas chez les sujets A et B.

L'exemple qui va suivre à présent nous montre une autre tentative de solution orientée vers la langue étrangère telle que l'entreprend le sujet $\mathrm{C}$ :

... ; um nach diesem späten Gast zu sehen, (3) ein später Gast, (2) un invité tard geht nicht, weil tard ja auf jeden Fall immer ein Adverb ist, [...], pour voir que était (2) cet invité, (4) hm, obwohl invité heißt zwar Gast, aber das hört sich hier doch komisch an, denn der hat den ja nicht eingeladen, dann kann man invité wohl nicht nehmen;...

Lorsque le sujet $\mathrm{C}$ se heurte à la difficulté de traduction à propos du mot Gast, qui n'en est une qu'en raison du contexte, la solution «invité» s'impose spontanément à lui. Toutefois, il met aussitôt en doute son exactitude et remarque à juste titre qu' «invité» en tant que forme substantivée du participe passé du verbe «inviter» ne peut s'utiliser que pour quelqu'un qui a été préalablement invité. Ce faisant, le sujet $\mathrm{C}$ oriente son processus mental vers la langue étrangère. À défaut d'une meilleure solution (il ne procède à aucune vérification dans le dictionnaire !), il finit pourtant par se décider pour cette solution (fausse !) :

...; (7) qui était cet homme vielleicht, (2) aber das ist auch schlecht, denn es muß ja nicht unbedingt ein Mann sein (3) ach nee, dann bleiben wir doch lieber bei invité;...

Ce processus de traitement mental fournit en même temps un exemple d'association. Lorsque le sujet C lit Gast dans le texte de départ, il fait immédiatement l'association avec «invité». Ce n'est qu'après avoir réfléchi à un autre problème, à savoir l'impossibilité de traduire l'adjectif spät par l'adverbe français «tard», qu'il met en doute la justesse de sa solution spontanée «invité». Toutefois l'association des deux mots, Gast et «invité» s'impose avec une telle force à l'esprit du sujet $\mathrm{C}$ que ce dernier n'a même pas l'idée de chercher d'autres solutions possibles dans le dictionnaire. Il se décide donc pour la solution «invité» bien qu'il ait reconnu, en orientant sa pensée vers la langue étrangère, que cette traduction était inadéquate dans le texte donné.

Dans ce cas l'association rend la solution plus difficile. Mais on rencontre chez les sujets de l'expérience aussi bien des exemples d'association facilitant la solution et qui influencent alors de façon positive le processus de traduction. Mais, dans le cadre qui nous est imparti, il nous faut renoncer à emprunter aux transcriptions d'autres citations illustrant ce phénomène.

D'intéressantes conclusions relatives aux processus mentaux de traitement intervenant au cours de la traduction peuvent être tirées des corrections auxquelles se livrent les sujets de l'expérience sur leur propre texte. D'une manière générale, il faut constater que les corrections sont relativement rares et, lorsqu'il y en a, elles sont le plus souvent apportées immédiatement, au beau milieu de la traduction d'une phrase : 
...; puis je pris mon bonnet en laine de la patère, le vieillard balançait un peu, (3) hm, nee, da müßte man dann ja auch balança nehmen, weil das ja auch so'ne Art Handlung von dem ist; le vieillard balança un peu quand je lui tirai le bonnet sur ses oreilles,...

Des corrections fondées sur la logique, comme dans cet exemple, restent toutefois plutôt une exception chez tous les sujets. Le plus souvent, ils se laissent guider par le sentiment; les sujets expriment alors un doute quant à la justesse ou à l'élégance des solutions trouvées, sans pour autant fonder cela de façon plus précise. La version est alors corrigée en fonction du fait qu'elle «sonne mieux», tout simplement. De telles décisions s'appuyant sur le sentiment sont particulièrement fréquentes face à des difficultés d'ordre lexical, difficultés qui sont, de loin, les plus fréquemment rencontrées.

À vrai dire, on pourrait s'attendre à ce que les sujets de l'expérience, après avoir traduit le texte entier et relu leur traduction une fois (ce qu'ils font tous les trois!), procèdent encore à un certain nombre de corrections. Or, il est étonnant de constater que c'est exactement le contraire qui se passe. Bien que certaines solutions fassent manifestement l'objet d'un doute au cours du processus même de la traduction, aucune correction n'est apportée à la fin. On peut en conclure que les solutions auxquelles les sujets ont abouti en traduisant restent relativement stables. On peut supposer que la disposition à (s'auto) corriger diminue en fonction du temps qui s'écoule après avoir trouvé une solution. Cela peut également provenir du fait qu'à la fin, les sujets sont simplement soulagés d'avoir assumé, d'une manière ou d'une autre, ce travail de traduction qui leur était imposé. En effet, tous les trois expriment leur soulagement de l'avoir enfin terminé.

La qualité du produit de leur traduction leur est absolument égale, comme le confirme expressément le sujet $\mathrm{A}$ :

je fais volontiers brûler la lumière devant la maison (5), tja, das ist wirklich nicht so schön; ich lasse gern das Licht vor dem Haus brennen (3), ja, wie kann man das schöner sagen? (10) Nee, wüßt' ich nicht, ich laß' das einfach so, ist auch egal.

La pause relativement longue de 10 secondes indique, certes, que le sujet $\mathrm{A}$ investit encore une fois son énergie mentale dans la recherche d'une solution plus élégante. Toutefois, cette énergie n'est, de toute évidence, plus assez forte pour l'amener à se servir des divers moyens de dépannage qu'il a à sa disposition. Naturellement, il est également pensable que les sujets n'attachent pas une bien grande importance à une «bonne» traduction puisqu'ils savent que leur travail ne sera pas noté (...ni n'aura aucune répercussion dans leurs études!). Seul le sujet $\mathbf{B}$ a une fois recours au dictionnaire pour une ultime correction. Celle-ci ne concerne toutefois pas une difficulté majeure de traduction ; il ne s'agit que de corriger le genre d'un mot dont il n'est pas sûr. Le sujet $\mathrm{C}$ ne procède à la fin qu'à une seule modification, et ce, en s'appuyant de toute évidence sur son sentiment.

En ce qui concerne l'utilisation des outils (de dépannage) mis à la disposition des sujets, il faut tout d'abord constater un fait assez surprenant : pas un seul des trois sujets de l'expérience n'a eu recours aux grammaires. Sans doute l'utilisation d'une grammaire ne leur semble-t-elle pas gratifiante pour aboutir à la solution d'une difficulté de traduction bien concrète, ou peut-être leur semble-t-elle trop compliquée, comme l'exprime une fois le sujet $\mathrm{C}$ :

...ja, aber die passé-simple-Form von rendre, (2) rendit? Ja, nee, müßt ich mal irgendwo nachschlagen, aber wo? (2) Ja, ich glaub', ich guck' erst mal im Petit Robert unter rendre nach, das geht schneller, als wenn ich die Konjugationen in der Grammatik suchen muß...

Lors de l'utilisation du dictionnaire, c'est la recherche d'une notion dans le dictionnaire bilingue allemand-français qui domine nettement. C'est à ce dictionnaire que les trois 
sujets ont très fréquemment recours pour trouver la solution d'une difficulté d'ordre lexical. Le dictionnaire bilingue français-allemand, de même que le dictionnaire (unilingue) français ne sont, par contre, utilisés que relativement rarement, soit pour la vérification d'hypothèses dans la langue cible, soit pour la vérification des notions proposées dans le dictionnaire allemand-français. C'est chez le sujet C que cette stratégie de vérification est la plus prononcée.

Lors de l'utilisation du dictionnaire bilingue allemand-français, il arrive que les sujets tombent sur une notion qu'ils connaissent comme mot de vocabulaire en soi, mais qui, sur le moment, ne leur est pourtant pas venue à l'esprit. Cela permet de conclure que l'information concernée est enregistrée dans la mémoire à long terme mais que, pour quelque raison que ce soit, elle n'a pu être activée dans la mémoire à court terme.

En ce qui concerne le vocabulaire totalement inconnu, pour lequel le dictionnaire bilingue offre plusieurs propositions comme autant de solutions possibles, la décision prise obéit souvent au sentiment, tout simplement. On trouve cette stratégie chez le sujet C qui - comme nous l'avons déjà évoqué - essaye par ailleurs, et de façon relativement fréquente, de vérifier son choix en s'appuyant sur les autres dictionnaires :

Ein wenig schwankte der Alte - le vieillard, (4) schwanken - tremblait? Nee, das paßt ja nicht, da muß ich auch noch mal nachschlagen (schlägt nach im deutsch-französischen Wörterbuch); schwanken, schwanken - chanceler, branler, balancer, vaciller, osciller, (3) hm, balancer klingt am besten, balancer; le vieillard balançait un peu,...

L'utilisation du dictionnaire bilingue allemand-français ne mène pas toujours à une solution satisfaisante. Souvent même, les sujets arrivent à une fausse solution en raison des propositions offertes par le dictionnaire et parce qu'ils procèdent à un choix en s'appuyant sur leur sentiment au lieu de s'assurer, à l'aide des autres dictionnaires à leur disposition, de la justesse de leur décision.

Pour finir, l'exemple suivant est tout particulièrement digne d'intérêt car il montre que le choix offert par le dictionnaire est parfois ressenti par le sujet plutôt comme un obstacle que comme un coup de pouce :

.... le vieux chancela un peu als - quand, (2) ich ihm, (3) ja, das ist dann Dativ, also quand je lui (4), hm, jetzt muß ich nachsehen, ob tirer auch ziehen in diesem Sinn heißen kann (sieht im deutsch-französischen Wörterbuch nach); tirer - o je, steht da viel (ist sichtbar abgeschreckt von der Länge des fast zwei Spalten umfassenden Eintrags), ziehen (beginnt zu lesen) - allgemein gleich tirer, (3) ach, was soll's, nehmen wir doch einfach tirer;...

Face au nombre impressionnant de propositions parmi lesquelles il doit effectuer son choix, le sujet B prend ici tout simplement le premier mot proposé, vraisemblablement en supposant qu'en fixant son choix sur la solution la plus «générale», il opte pour celle avec laquelle il court le moins de risque de se tromper. Toutefois, le sujet $\mathrm{B}$ ne semble pas non plus disposé ici à investir l'énergie mentale nécessaire pour aboutir à un choix fondé et garanti en s'aidant des propositions du dictionnaire.

Une des raisons qui permet de comprendre pourquoi aucun des sujets n'utilise les dictionnaires mis à leur disposition est vraisemblablement que, dans les cours de traduction de l'université, on n'apprend pas aux étudiants à se servir du dictionnaire, étant donné que son utilisation n'est pas autorisée lors des examens. On peut voir ici une raison supplémentaire pour non seulement autoriser l'utilisation d'ouvrages de références dans les cours de ce genre mais encore pour en faire l'objet d'un apprentissage et d'un entraînement systématiques.

Dans l'expérience étudiée, un transfert culturel pendant la traduction ne joue un rôle qu'à un seul moment, à savoir là où il s'agit de traduire le mot allemand Groschen. Nous avons là en effet une notion absolument ancrée dans la langue de départ et qui 
n'existe pas dans la langue cible. Parmi les trois sujets de l'expérience, seul le sujet $\mathrm{C}$ thématise en tant que telle la traduction de Groschen comme une difficulté. Les sujets A et $B$ se décident après un laps de temps relativement court pour une traduction par «centimes» ou «sous», remplaçant ainsi le concept de la langue de départ par un équivalent dans la langue cible; les pauses courtes indiquent qu'ils ne considèrent pas cette traduction comme un point de difficulté majeur. Le sujet C, lui, aussi, se décide relativement rapidement pour la solution «sous» :

..., un peu de charcuterie ou etliche Groschen, ja, Groschen kann man schlecht übersetzen,

(2) das müßte man dann vielleicht mit, (3) mit sous übersetzen, ou quelques sous aus der Hosentasche - de mes poches de pantalon?

L'idée de laisser tout simplement le mot allemand Groschen dans la traduction française en le plaçant entre guillemets n'est venue à l'esprit d'aucun des sujets.

Le peu d'importance pris par le transfert culturel dans l'expérience présente est tout simplement à mettre au compte du texte de départ allemand. Il ne contient que de rares notions propres à la langue de départ qui sont susceptibles de représenter une difficulté de traduction en raison de l'arrière-plan culturel des deux langues concernées. C'est à l'occasion de la traduction de textes de civilisation que les transferts culturels jouent vraisembablement un rôle bien plus important.

Pour conclure, jetons un regard sur les pauses que font les sujets de l'expérience lorsqu'ils pensent tout haut. À quelques légères différences près, il est permis de constater, de façon générale, que des pauses assez longues sont relativement rares. Le cas se présente lorsque les difficultés de traduction doivent être traitées avec un déploiement cognitif proportionnellement important.

Dans l'ensemble, et c'est là un résultat réjouissant, les trois sujets ne se sont pas soustraits aux règles de l'expérience en restant muets, bien que ce fût pour tous les trois la première expérience selon la méthode qui consiste à «penser à voix haute».

\section{INTERPRÉTATION GLOBALE DES RÉSULTATS ET CONCLUSION}

Malgré le caractère simplement exemplaire de l'évaluation, il ressort clairement de l'expérience réalisée que les processus mentaux, qui ont lieu au cours de la traduction, sont si divers et si individuellement marqués qu'ils ne se laissent ni représenter ni décrire par des modèles de portée générale. Les modèles mentionnés au début et mis sur pied par la traductologie ne prennent pas en considération les processus mentaux.

Essentiellement en raison du cadre qui nous est imparti, nous nous limitons ici à trois domaines dans lesquels les résultats documentés par cette étude se laissent interpréter. Ramenées à des formules concises, les façons de procéder des sujets se laissent caractériser de la manière suivantes :

Elles sont essentiellement :

- centrées sur le vocabulaire ;

- axées sur la difficulté présente ;

- orientées vers les expériences vécues.

Une part importante du travail mental des sujets apporte des éclaircissements sur l'apprentissage et le traitement mental du vocabulaire. On peut déduire ici que le recours au dictionnaire mental bilingue a essentiellement lieu sur la base de rapports d'équivalence interiorisés et fortement ancrés pour le vocabulaire de la langue étrangère. Ces rapports n'ont pas obligatoirement la qualité de rapports $1: 1$, mais sont toutefois relativement fixes. Et c'est cette rigidité qui bloque assez souvent l'activation de solutions dans le domaine lexical, qui, en fait, sont connues, mais qui sont différemment enregistrées mentalement. L'hypothèse défendue en psycholinguistique, selon laquelle les 
apprenants d'une langue étrangère disposent, eux aussi, d'un registre de données lexicales avec des inventaires différents spécifiques à chaque langue (voir à ce sujet quelques contributions dans Börner et Vogel 1994) trouve donc dans nos données une réelle confirmation. Cela dit, il semble qu'il en va ainsi : il n'est pas rare que la langue maternelle fonctionne comme une sorte de filtre sélectif pour la langue étrangère, filtre à travers lequel le recours mental à la langue étrangère est uniquement possible. Toutefois, nos données ne permettent pas de préciser s'il s'agit là d'un phénomène conditionné par la tâche à exécuter qui, alors, ne se manifesterait qu'en situation de traduction, ou bien s'il s'agit là d'un phénomène fondamental propre au traitement du savoir, à la construction du savoir, à l'acquisition de la langue et, enfin, propre à la production linguistique ayant lieu dans une langue étrangère. Des indices émanant d'autres travaux (par ex., Kern 1994 ; Wokusch 1994) tout comme la prise en compte d'observations fondamentales en provenance de la recherche sur le bilinguisme (voir, par ex., Königs 1994) permettent de conclure avec prudence que ce procédé n'est pas uniquement induit par la tâche à exécuter. Comme dans d'autres travaux déjà, il se révèle, dans les données présentées ici, que le domaine du vocabulaire domine totalement celui de la grammaire dans la concentration de l'activité mentale.

Le second résultat marquant qui, lui aussi avait pu être dégagé dans des travaux antérieurs, consiste en ce que les sujets, lorsqu'ils sont en train de traduire, utilisent beaucoup plus l'énergie mentale pour les difficultés rencontrées que pour les unités linguistiques qu'ils sont supposés maîtriser. Cela semble évident au premier abord, mais cela ne l'est que partiellement. D'une part, il est naturel que l'on doive s'investir plus pour ce dont on ne dispose pas déjà mentalement - la recherche d'un lexème qui n'est pas immédiatement disponible est naturellement plus astreignante que l'activation d'un élément ancré dans le dictionnaire mental. Mais on remarque, par ailleurs, que les processus de réception déjà intériorisés sont si fortement orientés vers les problèmes que, dans le cas extrême, ils tracent un chemin d'une difficulté à l'autre, chemin sur lequel la rencontre d'informations connues se trouve presque repoussée à l'arrière-plan. Dans ce cas, il n'est pas rare que cette façon de procéder, axée sur la difficulté, fasse dévier la perception pour des possibilités qui peuvent être dégagées de l'ensemble du texte et cela mène, ici et là, à des réalisations linguistiques erronées, même dans le cas d'unités linguistiques que le sujet maîtrise.

Comme troisième point, il faut mentionner que les sujets de l'expérience - de même que ceux des expériences précédentes - ont tendance à mettre au premier plan mental les problèmes qu'ils ont étudiés de façon intensive au cours de leur formation (voir plus haut à ce propos, l'accent mis sur l'utilisation des temps du passé lors de la première lecture du texte à traduire).

Dans l'ensemble, les résultats montrent - ceci est également une confirmation de résultats déjà acquis - un rôle considérable joué par l'individualité dans le processus de la traduction. Malgré toutes les différences individuelles, l'expérience a tout de même mis à nu quelques caractéristiques communes étonnantes dans les processus mentaux des sujets de l'expérience : il en est ainsi, par exemple, pour l'énorme restriction de l'activité de contextualisation qui ne s'effectue qu'au niveau de la phrase, voire même du syntagme; il en va ainsi de la disposition très réduite des sujets à des corrections ultérieures ou encore de l'utilisation insuffisante des moyens mis à leur disposition, tout particulièrement les dictionnaires. C'est précisément la constatation de cet usage déficient des trois sujets à propos des dictionnaires qui devrait inciter les responsables de la formation universitaire en traduction à réfléchir. Étant donné que - comme il en a déjà été fait la supposition - i, il est pensable que les étudiants n'ont jamais vraiment appris à se servir d'un dictionnaire, faute d'occasions, on devrait en tenir compte dans l'organisation des 
études. L'utilisation des dictionnaires devrait faire l'objet d'une formation systématique dans les cours de traduction. Dans les situations d'examen - et naturellement, de manière conséquente également lors de l'examen final - il devrait être permis à tous les candidats d'avoir recours au moins à un dictionnaire français unilingue. Si tous les candidats à l'examen disposent du même ouvrage, cela est fort compatible avec le but visé par l'examen. Une autre leçon didactique à en tirer consisterait à exiger un entrâ̂nement systématique à diverses stratégies, et à susciter une (auto)réflexion continuelle sur sa propre façon de procéder mentalement. En parlant de stratégies, il n'est pas question ici de méthodes dans le sens adopté par la traductologie, mais de procédés mentaux intervenant dans la résolution de difficultés mentales. Dans le domaine de l'acquisition des langues étrangères en milieu institutionnel, de telles considérations jouent déjà un rôle de plus en plus grand. La didactique de la traduction, en tant que branche de la traductologie, devrait, à son tour, se préoccuper de telles considérations et ne pas démissionner. Pour des apprenants se formant à la traduction ainsi que pour des apprenants dans le domaine des langues étrangères, il existe, selon toute vraisemblance, une quantité non négligeable de problèmes communs dans le domaine de l'activité mentale, problèmes dont la solution devrait être recherchée, peut-être également à travers un effort commun de la part des représentants des domaines de recherche concernés. Somme toute, il se pourrait que ces deux domaines soient beaucoup plus proches l'un de l'autre que de nombreux responsables ne veulent — un peu trop rapidement — l'admettre.

Note

1. Tous nos remerciements à Babett Parent pour son aide linguistique lors de la rédaction de cet article.

\section{RÉFÉRENCES}

BELL, R. T. (1991) : Translation and Translating. Theory and Practice, London/New York, Longman.

BÖRNER, W. et K. VOGEL (dir.) (1994): Kognitive Linguistik und Fremdsprachenerwerb. Das mentale Lexikon, Tübingen, Narr.

GARDES, J.-C., LACOURIÈRE, C. et J.-P. MASQUELIEZ (1983) : Übersetzungskurs für Anfänger und Fortgeschrittene (Deutsch-Französisch), deuxième édition, Borken.

GARDES, J.-C., LACOURIÈRE, C. et J.-P. MASQUELIEZ (1983) : Lösungsheft zum Übersetzungskurs für Anfänger und Fortgeschrittene (Deutsch-Französisch), deuxième édition, Borken.

GERLOFF, P. (1986) : «Second Language Learner's Reports on the Interpretive Process: Talk-Aloud Protocols of Translation», J. House et S. Blum-Kulka (dir.), Interlingual and Intercultural Communication. Discourse and Cognition in Translation and Second Language Acquisition Studies, Tübingen, Narr, pp. 243-262.

HÖNIG, H. G. (1988) : «Wissen Übersetzer eigentlich, was sie tun?», Lebende Sprachen, 1, pp. 10-14.

KERN, R. G. (1994): «The Role of Mental Translation in Second Language Reading», Studies in Second Language Acquisition, 16-4, pp. 441-461.

KOLLER, W. (1992) : Einführung in die Übersetzungswissenschaft, quatrième édition, Heidelberg, Quelle \& Meyer.

KÖNIGS, F. G. (1986) : «Der Vorgang des Übersetzens: Theoretische Modelle und praktischer Vollzug. Zum Verhältnis von Theorie und Praxis in der Übersetzungswissenschaft», Lebende Sprachen, 1, pp. 5-12.

KÖNIGS, F. G. (1987) : «Was beim Übersetzen passiert. Theoretische Aspekte, empirische Befunde und praktische Konsequenzen», Die Neueren Sprachen, 2, pp. 162-185.

KÖNIGS, F. G. (1988) : «Auf der Suche nach dem richtigen Wort. Analysen zum lexikalischen Suchverhalten beim Schreiben in der Fremdsprache und beim Hinübersetzen», Fremdsprachen Lehren und Lernen, 17, pp. 99-117.

KÖNIGS, F. G. (1989) : «Übersetzungsdidaktik und Psycholinguistik. Gedanken und Befunde zu einer ebenso zwangsläufigen wie notwendigen Verbindung», F. G. Königs (dir.), Übersetzungswissenschaft und Fremdsprachenunterricht. Neue Beiträge zu einem alten Thema, München, Goethe-Institut, pp. 147-178.

KÖNIGS, F. G. (1990a) : Beim Übersetzen schreibt man - übersetzt man auch beim Schreiben? Ein psycholinguistisch orientierter Vergleich zweier fremdsprachlicher Produktionsprozesse bei fortgeschrittenen deutschen Spanischlernern, thèse d'État, Universität Bochum.

KÖNIGS, F. G. (1990b) : «Wie theoretisch muß die Übersetzungswissenschaft sein? Gedanken zum TheoriePraxis-Problem», Taller de Letras, 18, pp. 103-120. 
KÖNIGS, F. G. (1994) : “'Chacun à son goût ?' Zur Rolle der Muttersprache im Fremdsprachenunterricht aus der Sicht der Sprachlehrforschung», Landesspracheninstitut NRW-Arabicum Bochum, Arabischunterricht in Deutschland. Bewährte Ziele, neue Perspektiven. Vorträge der 4. Arabischlehrerkonferenz in Bochum, 18.-20. Juni 1993, Bochum, Landesspracheninstitut, pp. 31-61.

KRINGS, H. P. (1986): Was in den Köpfen von Übersetzern vargeht. Eine empirische Untersuchung zur Struktur des Übersetzungsprozesses an fortgeschrittenen Französischlernern, Tübingen, Narr.

LÖRSCHER, W. (1991): Translation Performance, Translation Process, and Translation Strategies. A Psycholinguistic Investigation, Tübingen, Narr.

NEWMARK, P. (1991) : About Translation, Clevedon, Multilingual Matters.

SMITH, V. (1994) : Thinking in a Foreign Language. An Investigation into Essay Writing and Translation by L2 Learners, Tübingen, Narr.

STOLZE, R. (1994) : Übersetzungstheorien. Eine Einführung, Tübingen, Narr.

TIRKKONEN-CONDIT, S. (dir.) (1991): Empirical Research in Translation and Intercultural Studies, Tübingen, Narr

TIRKKONEN-CONDIT, S. et J. LAFFLING (dir.) (1993) : Recent Trends in Empirical Translation Research, Joensuu.

WOKUSCH, S. (1994): 'Man hat nicht mehr diese Angst des Unbekannten'. Aspekte des Erwerbs und der Verwendung einer Fremdsprache mit Beispielen aus dem Gebrauch des Deutschen durch frankophone Lernende, Bochum, Brockmeyer.

\section{ANNEXE}

Texte à traduire :

\section{Der Bettler}

Einmal im Winter, an einem stürmischen Abend, klopfte es an der Tür. Bei solchem Wetter lasse ich gern das Licht vor dem Haus brennen, damit mir die Nacht nicht zu nah an die Fenster kommt. Nun ging ich also verdrossen, um nach diesem späten Gast zu sehen. Der Wind riß mir gleich die Klinke aus der Hand. Draußen stand ein alter Mann auf den Stufen, ich kannte ihn. Er kam oft vorüber, klopfte und hielt mir die Hand entgegen. Nie sagte er ein Wort des Grußes oder des Dankes, er sah mich nur an mit seinen wäßrigen Trinkeraugen, und ich gab ihm, was mir einfiel, ein Endchen Wurst oder etliche Groschen aus der Hosentasche. Über der Schulter trug er einen Stock und daran hing ein Sack. Aber was mich jetzt ärgerte, war sein kahler Kopf, es lag ihm wahrhaftig schon Schnee auf dem Schädel. Da nahm ich meine wollene Haube vom Haken, ein wenig schwankte der Alte, als ich ihm die Mütze über die Ohren zog, und dann ging er wortlos davon.

Das aber war der Augenblick, in dem ich mich hätte besinnen müssen. Ich hätte an die rückwärtige Kammer denken sollen, o ja, ich dachte auch daran. Dort stand ein leeres Bett bereit. Aber zugleich dachte ich an mein sauberes Haus, wie dieser Kerl seine Fetzen auf den gewachsten Boden fallen ließe und unter das frische Leintuch kröche, mit seinen Läusen. Ünd da schlug ich die Tür zu. nach Karl Heinrich Waggerl

source: Gardes/Lacourière/Masqueliez, Übersetzungskurs für Anfänger und Fortgeschrittene (Deutsch-Französisch), deuxième édition, Borken 1983, p. 96.

Traduction proposée :

\section{Le mendiant}

Une fois, en hiver (par) un soir de tempête, on/quelqu'un frappa à la porte. Par un temps pareil j'aime laisser/je laisse volontiers la lumière allumée devant la maison pour que la nuit ne s'approche pas trop près de mes fenêtres. Contrarié, j'allai donc voir qui était ce visiteur/cet hôte, tardif. Tout de suite le vent $m$ 'arracha des mains la poignée de la porte. Dehors, sur les marches (de l'escalier) se tenait/se trouvait un vieil homme, je le connaissais. Il passait souvent (par) ici, frappait (à la porte) et (me) tendait la main/tendait la main vers moi. Jamais il ne disait une parole (en guise) de salutation ou de remerciement, il me regardait seulement/il ne faisait que me regarder de/avec ses yeux humides de buveur, et je lui donnais ce qui me venait à l'esprit/l'idée, un (petit) bout/morceau de saucisson ou quelques sous tirés de ma poche de pantalon. Sur l'épaule il portait un bâton auquel était suspendu/accroché un sac. Mais ce qui m'agaça alors/à ce moment-là, ce 
fut/c'était sa tête nue/chauve, il avait déjà bel et bien de la neige sur le crâne. Alors je décrochai mon bonnet de laine, le vieux (bonhomme) vacilla/chancela un peu lorsque je lui passai le bonnet sur les oreilles, et puis il partit/s'en alla sans mot dire/sans dire un mot. Mais c'était/ce fut le moment où j'aurais dû réfléchir un peu. J'aurais dû penser à la pièce/chambre de derrière, oh oui, j'y ai bien pensé. Il y avait là un lit (de) vide, tout prêt. Mais en même temps je pensai(s) à ma maison toute propre, à ce gars qui laisserait tomber ses haillons/guenilles sur le sol ciré et qui se glisserait sous le drap de lin fraîchement lavé/tout propre, lui et ses poux. Et alors je claquai la porte.

source : Gardes/Lacourière/Masqueliez, Lösungsheft zum Übersetzungskurs für Anfänger und Fortgeschrittene (Deutsch-Französisch), deuxième édition, Borken 1983, p. 138 f.

Penser à haute voix, sujet $\mathrm{A}$ :

(Prend le texte et commence à le lire) Einmal im Winter, an einem stürmischen Abend, klopfte es an der Tür (2) - passé simple. Bei solchem Wetter lasse ich gern das Licht vor dem Haus brennen, damit mir die Nacht nicht zu nah an die F... (hésite), damit mir die Nacht nicht zu nah an die Fenster kommt. Nun ging ich also verdrossen, um nach diesem späten Gast zu sehen (2) - auch passé simple. Der Wind riß mir gleich die Klinke aus der Hand. Draußen stand ein alter Mann auf den Stufen, ich kannte ihn - éventuel imparfait. Er kam oft voriber, klopfte und hielt mir die Hand entgegen (3) - Hintergrundbeschreibung, also imparfait. Nie sagte er ein Wort des Grußes oder des Dankes, er sah mich nur an mit seinen wäßrigen Trinkeraugen, und ich gab ihm, was mir einfiel, ein Endchen Wurst oder etliche Groschen aus der Hosentasche - weiterhin imparfait. Über der Schulter trug er einen Stock und daran hing ein Sack - auch imparfait. Aber was mich jetzt ärgerte, war sein kahler Kopf, es lag ihm wahrhaftig schon Schnee auf dem Schädel (3) - weiterhin imparfait noch. Da nahm ich meine wollene Haube vom Haken, ein wenig schwankte der Alte, als ich ihm die Mütze über die Ohren zog, und dann ging er wortlos davon (2) - das ist jetzt passé simple, weil die Handlung einsetzt. Das aber war der Augenblick, in dem ich mich hätte besinnen müssen (6) - auch passé simple. Ich hätte an die rückwärtige Kammer denken sollen, o ja, ich dachte auch daran. Dort stand ein leeres Bett bereit. Aber zugleich dachte ich an mein sauberes Haus, wie dieser Kerl seine Fetzen auf den gewachsten Boden fallen ließe und unter das frische Leintuch kröche, mit seinen Läusen. Ünd da schlug ich die Tür zu.

Da muß ich jetzt aber viele Vokabeln nachgucken (5). Hm, jetzt fang ich einfach mal an. Der Bettler - le mendiant (15). Einmal im Winter (5), une fois en hiver (9), an einem stiurmischen Abend (2), was heißt das jetzt ? (2) Stürmisch, irgendwas mit tourmet (4), nee, stürmisch guck' ich nach (prend le dictionnaire allemand-français); stürmisch — tempétueux, orageux, démonté, agité, houleux (3) ; orageux ist das, was mir am geläufigsten ist, deswegen nehme ich das mal. Une fois en hiver (3), hm (2), un soir orageux (10), klopfte es an der Tür (5), ja, klopfen guck' ich auch nach (prend le dictionnaire allemand-français); klopfen - frapper, battre, palpiter, cogner, hm (continue à chercher dans le dictionnaire), battre, taper (3), ja, nehmen wir mal taper. Also, une fois en hiver, un soir orageux, hm (3), il tapa (3), ja, passé simple, hm (2), il tapa à la porte (3). Bei solchem Wetter lasse ich gern das Licht vor dem Haus brennen (15), hm, quand il fait (10) du mauvais temps (13), lasse ich gern das Licht vor dem Haus brennen (6), hm, brennen lassen heißt (3) faire brûler vielleicht (4), je fais volontiers (5), hm, je fais volontierts brûler (13), nein, das ist nicht richtig, das heißt faire kann man hier nicht gebrauchen (7), hm (stöhnt), (8) vielleicht mit allumer, nee, geht auch nicht (7), hm, ach, ich laß das so; je fais brûler, ach nein, je fais volontiers brûler (16), hm, ja, man kann nicht einfach la lumière sagen, das geht nicht (5), vielleicht le feu (3), nein, das ist auch nicht gut (2), dann lassen wir trotzdem lieber lumière (2), ja, la lumière (4) devant la maison (7), damit mir die Nacht nicht zu nah an die Fenster kommt (4). Quand il fait du mauvais temps, je fais volontiers brûler la lumière devant la maison, damit mir die Nacht nicht zu nah an die Fenster kommt (6). Hm, damit - pour que (5), hm, die Nacht (5), das muß irgendwie umschrieben werden, das kann man nicht genauso sagen (4), la nuit (6), $\mathrm{hm}$, vielleicht mit pour que la nuit (7) ne s'approche (3), hm, pas trop (6), hm (4), à la fenêtre (3). Nun ging ich also verdrossen, um nach diesem späten Gast zu sehen (2). Hm, was heißt denn verdrossen? (4) Das weiß ich überhaupt nicht, das muß ich nachgucken (sieht im deutsch-französischen Wörterbuch nach); verdrossen das kann man mit fâché übersetzen, oder mit contrarié ; (3) nehm' ich mal fâché (4), hm (3), nun ging ich also verdrossen (9), hm, das meint hier wohl eher 'ne zeitliche Abfolge, deswegen sag' ich 
hier (3), hm, puis (3) j'allai - passé simple - (2) fâché (5), um nach diesem späten Gast zu sehen. (7) Hm, nach jemandem sehen (6), vielleicht nach jemandem Ausschau halten (5), hm, nach jemandem sehen (3), ja, regarder ist das auf jeden Fall nicht (11), vielleicht pour aller voir, aber nee, das heißt doch mehr besuchen (8), vielleicht um zu sehen, ob der späte, einfach ob der Gast kommt (2); also, pour aller (3), nein, pour voir (9), si (14), hm, ja Gast, das guck' ich am besten noch mal nach (sieht im deutsch-französischen Wörterbuch nach) ; (5) ja, für Gast nehm' ich am besten hôte ; pour voir si (2), hm, mon hôte (6), si mon hôte venait (12). Der Wind riß mir gleich die Klinke aus der Hand (4), hm, das muß man irgendwie umschreiben (5), le vent (3) était si fort (10), ach, wie kann ich das sagen, die Klinke aus der Hand reißen? Le vent était si fort (5), das umschreib' ich (3), que ma main ne pouvait pas (3), hm, Klinke, das guck' ich lieber mal nach (regarde dans le dictionnaire allemand-français); ach ja, Klinke heißt la poignée ; also (4), le vent était si fort que ma main ne pouvait pas (4) tenir la poignée (7), hm, ja. Draußen stand ein alter Mann auf den Stufen (7) ; draußen heißt dehors (5), un vieux homme (3) stand auf den Stufen (10), vielleicht était debout (5), ja Stufe muß ich nachgucken (regarde dans le dictionnaire allemand-français) ; ach, la marche, das hätt' ich auch wissen müssen ; draußen stand ein alter Mann auf den Stufen, (5) dehors, un vieux homme (4), était debout (5), heißt das wohl sur les marches? oder dans les marches ? (6) Ja, jetzt muß man die richtige Präposition wissen (8), ja, sur les marches sag' ich mal, ich bin mir aber nicht sicher (5); ich kannte ihn - je le connaissais (7). Er kam oft voriber, klopfte und hielt mir die Hand entgegen (6). Ja, vorüberkommen (2), vielleicht mit passer (5) ; il passait (2) souvent (3), klopfte und hielt mir die Hand entgegen (4). Klopfen hatten wir doch oben (cherche le passage dans le texte), ach ja, taper; hm (3), tapait (2), ja, sicher, das muß imparfait sein; il passait souvent, tapait und hielt mir die Hand entgegen (5). Hm, die Hand entgegenhalten, hm (7), il me donna la main ? (3) Donna, donnait, nee, donnait natürlich, il me donnait la main (5). Nie sagte er ein Wort des Grußes oder des Dankes (6). Also (5), il ne disait jamais (3) ein Wort des Grußes (7), tja, wie kann man das denn sagen, ein Wort des Grußes (11), ja, ob man das so sagen kann, ein Wort des Grußes oder des Dankes? (5) Hm, il ne disait jamais des mots de salut? (7) Nein, ich guck' Gruß erst noch mal genau nach (cherche dans le dictionnaire allemand-français) ; ja, was haben wir denn da ? Salut, salutation, compliments, respects, hommages (2), nein ; Grüße bestellen - transmettre des amitiés (2), nein; ja, vielleicht des mots de salut, de salutation (8), hm (5), nein; oder ich umschreib' das einfach (4), il ne disait jamais (6), hm, (5), nein, il ne disait jamais (2) un mot de salutation, obwohl das bestimmt falsch ist, aber ich schreib' das jetzt einfach mal hin (zögert aber noch) ; (10) Nein, genau, ich mach' das anders, (5), il ne disait jamais 'je vous salue' (8) ou, hm (4), 'merci' (5) ; er sah mich nur an mit seinen wäßrigen Trinkeraugen (3), hm, er sah mich an - il me regardait (6), ja, das ist auch noch imparfait, weil's immer passiert; er sah mich nur an (2), il me regardait, nur, hm (3), mit seinen wäßrigen Trinkeraugen (5), ja das müßte ich erst mal nachgucken (3); nein, ich guck' das nicht nach, ich werd' das lieber umschreiben (3); hm (2), il me regardait (2) de ses yeux (4), hm (7), ses yeux d'un ivrogne (6), ja wäßrig, das guck' ich doch besser nach (cherche dans le dictionnaire allemand-français); wäßrig - aqueux, feucht humide, ja humide kenn' ich, das wird ja wohl richtig sein ; (2) ja genau, il me regardait de ses yeux d'un ivrogne, très humides (3), ja ; und ich gab ihm, was mir einfiel, ein Endchen Wurst oder etliche Groschen aus der Hosentasche (5). Ünd ich gab ihm (2), hm (3), ja, entweder rendre oder donner (2), ja donner ist besser ; also, und ich gab ihm - et je lui donnais (4), hm, was mir einfiel (5), hm (16), vielleicht irgendwas mit imaginer (8), et je lui donnais ce qui (2), nee, ce que (5), hm (4), ce que j'imaginais (3); ein Endchen Wurst (13), tja (2), la fin d'un saucisson (4), oder saucisse? (2) Das guck' ich noch mal nach (cherche dans le dictionnaire allemand-français); so, was haben wir denn hier? Saucisse - Wurst zum Heißmachen oder Braten (2), ja das ist wohl das Richtige ; also, la fin d'une saucisse (2) oder etliche Groschen aus der Hosentasche. Hm (4), la fin d'une saucisse ou (3), hm (5), beaucoup de centimes (4), ja, beaucoup de centimes de ma poche (2) de pantalon (3). Über der Schulter trug er einen Stock und daran hing ein Sack (6). Puh (3), hm (7), tragen, ja (2), tragen - porter geht eigentlich hier nicht so gut, das ist mehr für Kleider (10); hm, ja, auch die Präposition ist schwierig (5); über der Schulter trug er einen Stock (15), hm, was nehm' ich denn da ? (10) Vielleicht tenir, tenir un bâton (7); also, (9) il tenait un bâton (2), puh (2), über der Schulter (5), vielleicht au-dessus (3), das ist eigentlich nicht so gut (3), na ja, au-dessus de ses épaules (5), und daran hing ein Sack (8). Hm (3), ja, da mach' ich 'nen neuen Satz 'raus, aber hängen muß ich erst mal nachsehen (cherche dans le dictionnaire allemand-français); hängen - 
suspendre (2), auf einen Haken - accrocher (2), oder einfach pendre? (3) Nee, vielleicht accrocher (2), ja, das nehmen wir mal; also, (8) un sac (3), un sac accrochait (2), hm (5), un sac accrochait, ja die Präposition (4), un sac accrochait (3) au bâton (10), hm (7). Aber was mich jetzt ärgerte (3), ja, ce qui me f... (12), hm (3), was sein kahler Kopf, es lag ihm (11), hm (7), nee, das sag' ich anders ; (3) mais j'étais fâché (4), hm (2) à cause ... (5); das ist eigentlich jetzt aber passé simple, oder nicht? (2) Was mich jetzt ärgerte, war sein kahler Kopf, es lag ihm wahrhaftig schon Schnee auf dem Schädel. Da nahm ich meine wollene Haube vom Haken ... (5) Nee, da oben, das ist noch imparfait ; mais j'étais fâché à cause (3), hm (4), kahlköpfig, das heißt doch chauve, oder ? (5) Mais j'étais fâché à cause de sa tête chauve (4), hm (7), es lag ihm wahrhaftig schon Schnee auf dem Schädel (lacht), (3) puh, (4) tja, (5), das ist ein Bild, aber kann man das genauso wiedergeben ? (5) $\mathrm{Hm}$ (3), vielleicht mit es schien (5); il semblait que de la neige (5), hm (2), était (3), Schädel heißt crâne, le crâne, ja (3), sur son crâne (5) ; oder nur le crâne ? (5) Nee, nur son crâne ist gut, was soll's (7). Da nahm ich meine wollene Haube vom Haken (7), hm (8), da nahm ich meine wollene Haube vom Haken, ein wenig schwankte der Alte, als ich ihm die Mütze über die Ohren zog, und dann ging er wortlos ... (3). Hm, vielleicht dans ce moment (6). Da nahm ich meine wollene Haube vom Haken (2), ja, wollene Haube muß ich nachgucken (regarde dans le dictionnaire allemand-français) ; ach natürlich, wollen heißt de laine, oder en laine (3), vom Haken (2), puh, Haken muB ich auch nachgucken (cherche dans le dictionnaire allemand-français); Haken - le crochet (3), vom Haken nehmen - décrocher (4), einen Haken schlagen, das ist es nicht (3), dann nehm' ich décrocher ; dans ce moment, (2), da nahm ich meine wollene Haube vom Haken, ja, ja, dans ce moment, je décrochai (6), ja, passé simple, je décrochai (2), ach so, ja, die Haube, das weiß ich auch nicht (cherche dans le dictionnaire allemand-français); Haube - le bonnet, ja das hab' ich öfter schon mal gehört, ja ja, das stimmt (2) ; also, dans ce moment, je décrochai (2) mon bonnet de laine (2), hm, ein wenig schwankte der Alte (4), hm (3), was kann denn schwanken heißen ? (2) Tourner vielleicht (3), le vieux tourna un peu (4), als ich ihm die Mütze über die Ohren zog (5). Hm (2), dans ce moment, je décrochai mon bonnet de laine, le vieux tourna un peu (3), als ich ihm (5), $\mathrm{hm}$, mit gérondif geht das nicht (2), also lorsque (7), als ich ihm die Mütze über die Ohren zog (2), über die Ohren ziehen (3), (stöhnt) vielleicht steht das bei Mütze (cherche dans le dictionnaire allemand-français sous. le mot 'Mütze') ; nein, bei Mütze steht das nicht, da steht nur la casquette oder le béret (3), la casquette, ja, aber was machen wir damit ? Als ich ihm die Mütze über die Ohren zog - lorsque, ja (5), über die Ohren ziehen (4), tja, tirer geht auf keinen Fall (19), (stöhnt) guck' ich mal nach (cherche dans le dictionnaire allemand-français); also, tirer nicht (2), herausziehen - sortir oder extraire, (2) tracer, hm (7), mal weitergucken, (3) das Fell uiber die Ohren ziehen écorcher (2), aber das ist doch was anderes (3), das meint das ja hier wörtlich (10), (continue $\grave{a}$ chercher) vielleicht trainer (5), oder doch tirer? (2) Machen wir's doch mit tirer, was soll's. Also, lorsque je tirai (2); le vieux tourna un peu, lorsque je tirai (3), hm, passé simple, ja, die Mütze über die Ohren zog (3), lorsque je tirai la casquette (24), hm, das meint das hier doch so, daß (2), daß der die dann aufgesetzt kriegt (3), also lorsque je donnai, je lui donnai la casquette, ja, das könnte man wohl besser sagen (9), (stöhnt), ja, lorque je lui donnai la casquette (3), und dann ging er wortlos davon. (3) Puis (2), hm (4), il s'en alla (5) sans dire mot (2), ja. Das aber war der Augenblick, in dem ich mich hätte besinnen müssen. (5) Ce fut (2), ja, passé simple nehm' ich, le moment (3), hm (2), in dem ich mich hätte besinnen müssen (3), hm, sich besinnen (2), da muß ich mal überlegen ; ce fut le moment (2) où (16), hm, was ist das denn überhaupt hier für'ne Zeit ? (5) Ich hätte mich besinnen müssen (2), ce fut le moment, hm (4), où j'aurais dû réfléchir ? (8) Ja, où j'aurais dû réfléchir (3). Ich hätte an die rückwärtige Kammer denken sollen (6), ja, das sind alles ganz normale Konditionalsätze, das ist glaub' ich so in Ordnung; ich hätte an die rückwärtige Kammer denken sollen, o ja, ich dachte auch daran. Häh, welche rückwärtige Kammer denn ? (4) Ach so (2), ah ja, das Zimmer liegt hinten im Haus (3); also, il aurait fallu (2) penser (3) à la pièce, hm (5), an die rückwärtige (6), à la pièce en arrière, ja (2); o ja, ich dachte auch daran, hm (5) vielleicht mit maintenant (3), hm (4), j'y pensai (6), ja. Dort stand ein leeres Bett bereit (3), là-bas (3), oder dans cette pièce ? (5) Nee, là-bas kann ich ruhig sagen, là-bas stand ein leeres Bett bereit. (6) $\mathrm{Hm}$, bereitstehen (4), un lit (3) était libre (2), ja, das ist gut. Aber zugleich dachte ich an mein sauberes Haus (2), hm (3), cependant, oder ? (2) Aber zugleich (3), vielleicht (4), au, nee, dans le même moment ; cependant, dans le même moment (20), nee, warte mal, davor hatte ich passé simple (3), jetzt ist das aber imparfait, das ist 'ne Hintergrundbeschreibung; cependant, dans le même moment, je pensais 
(2) à ma maison propre (3), ja, nachgestellt heißt das sauber, wie dieser Kerl seine Fetzen auf den gewachsten Boden fallen ließe (4), hm, mit wie ist das immer so'ne Sache (5) ; also, wie dieser Kerl (3), ja, comment würd' ich da sagen (4), comment ce mec (3), ja, das ist ja hier eher abwertend (2), seine Fetzen, das weiß ich nicht, auf den gewachsten Boden fallen ließe (4), hm, also gucken wir erst mal Fetzen nach (sieht im deutsch-französischen Wörterbuch nach); Fetzen - lambeau, chiffon (stutzt) ; Lumpen - haillon (2), ja, das wird das ja hier wohl sein (2), also ses haillons ; je pensais à ma maison propre (2), hm (2), comment ce mec (10), et comment ce mec faisait tomber ses haillons (14), nee, tomber ist auch nicht das Passende, aber das weiß ich nicht anders (3); comment ce mec faisait tomber ses haillons sur le (2), hm (4), gewachsten Boden, das guck' ich lieber nach; erst mal gewachst nachgucken (cherche dans le dictionnaire allemand-français); also wachsen, das heißt cirer, ach ja (8), ja (se rend compte en lisant les informations sous le mot 'Wachs', que la traduction de 'Bohnerwachs' est 'cire à parquet'), vielleicht auch mit parquet (2), ja, le parquet ciré, comment ce mec faisait tomber ses haillons au parquet ciré (2) und unter das frische Leintuch kröche, mit seinen Läusen (5), ja, ou comment il (12), hm, kriechen unter (23), hm, ja, das Leintuch heißt auf jeden Fall le drap, le drap propre (14), kriechen (3), vielleicht einfach mit se coucher, comment il se, hm (3), couchait (4) dans (2), ja, dans (2) vielleicht mon, ja, mon drap propre (3) mit seinen Läusen, hm, das muB ich auch noch nachgucken (cherche dans le dictionnaire allemand-français); Laus - le pou, ja, le pou ist die Laus, also avec ses poux ; comment il se couchait (2), hm, ja, vielleicht hier einbauen (4), comment il se couchait avec ses poux (3), oder plein de poux (5), nein, avec ses poux ist besser, also avec ses poux dans mon drap propre (3). Und da schlug ich die Tür zu. Hm (5), ja, puis, puis je fermai (2), ja, passé simple, la porte (3). Puh, fertig ! Jetzt muß ich mir das alles noch mal durchlesen.

(Le sujet A lit sa traduction, mais il ne corrige rien. Les temps choisis sont expressément approuvés. Par deux fois, A doute de l'option choisie, mais comme il ne trouve pas d'autres solutions plus adaptées, il renonce à corriger son texte.)

(...) je fais volontiers brûler la lumière devant la maison (5), tja, das ist ja wirklich nicht so schön ; ich lasse gern das Licht vor dem Haus brennen (3), ja, wie kann man das schöner sagen? (10) Nee, wüßt' ich nicht, ich laß' das einfach so, ist auch egal.

Le vent était si fort que ma main ne pouvait pas tenir la poignée (6), tja, tenir ist vielleicht auch nicht richtig (5), aber ich weiß nichts anderes, das muß ich auch so lassen.

(temps $=62$ minutes)

Traduction faite par A :

\section{Le mendiant}

Une fois en hiver, un soir orageux, il tapa à la porte. Quand il fait du mauvais temps je fais volontiers brûler la lumière devant la maison, pour que la nuit ne s'approche pas trop à la fenêtre. Puis j'allai fâché pour voir se mon hôte venait. Le vent était si fort que ma main ne pouvait pas tenir la poignée. Dehors, un vieux homme était debout sur les marches. Je le connaissais. Il passait souvent, tapait et me donnait la main. Il ne disait jamais 'je vous salue' ou 'merci', il me regardait de ses yeux d'un ivrogne, très humides, et je lui donnais ce que j'imaginais, la fin d'une saucisse ou beaucoup de centimes de ma poche de pantalon. Il tenait un bâton au-dessus de ses épaules. Un sac accrochait au bâton. Mais j'étais fâché à cause de sa tête chauve; il semblait que de la neige était sur son crâne. Dans ce moment, je décrochai mon bonnet de laine, le vieux tourna un peu lorsque je lui donnai la casquette. Puis il s'en alla sans dire mot. Ce fut le moment où j'aurais dû réfléchir. Il aurait fallu penser à la pièce en arrière, maintenant j'y pensai. Là-bas, un lit était libre. Cependant, dans le même moment, je pensais à ma maison propre et comment ce mec faisait tomber ses haillons au parquet ciré ou comment il se couchait avec ses poux dans mon drap propre. Puis je fermai la porte. 